\title{
Second-order polarization-mode dispersion in photonic crystal fibers
}

\author{
Larsen, T; Bjarklev, Anders Overgaard; Peterson, A; Folkenberg, J
}

Published in:

Optical Fiber Communications Conference, 2003. OFC 2003

Publication date:

2003

Document Version

Publisher's PDF, also known as Version of record

Link back to DTU Orbit

Citation (APA):

Larsen, T., Bjarklev, A. O., Peterson, A., \& Folkenberg, J. (2003). Second-order polarization-mode dispersion in photonic crystal fibers. In Optical Fiber Communications Conference, 2003. OFC 2003 (Vol. 1). IEEE.

\section{General rights}

Copyright and moral rights for the publications made accessible in the public portal are retained by the authors and/or other copyright owners and it is a condition of accessing publications that users recognise and abide by the legal requirements associated with these rights.

- Users may download and print one copy of any publication from the public portal for the purpose of private study or research.

- You may not further distribute the material or use it for any profit-making activity or commercial gain

- You may freely distribute the URL identifying the publication in the public portal

If you believe that this document breaches copyright please contact us providing details, and we will remove access to the work immediately and investigate your claim. 
While the polarization decorrelation length is the scale on which the ensemble average

$\left\langle S_{1}(z)\right\rangle$

reaches its asymptotic value of 0 , the diffusion length $d_{k}$ is defined as the distance it takes for the variance of the Stokes parameter $S_{k}$ to reach its asymptotic value of $1 / 3$. This asymptotic value is $1 / 3$ since the Stokes vectors are asymptotically uniformly distributed on the Poincaré sphere. We define the diffusion length $d$ to be the maximum of the values $d_{1}, d_{2}$, and $d_{3}$. In Fig. 2, we show the results of simulations for different values of $\tau / \sigma$. These results show that as the ratio $\tau / \sigma$ converges to the isotropic value 1 , the local diffusion length becomes proportional to the fiber decorrelation length $h_{\text {fiber }}$ However, the diffusion length has the same convex dependence on $h_{\text {fiber }} / L_{B}$ for all values of $\tau / \sigma$, except for the isotropic case $\tau / \sigma=$ 1 , which is a singular limit. Thus, a small ellipticity has no significant effect on system behavior as compared to the case of only linear birefringence. However, the variation in the diffusion lengths for small $h_{\text {fiber }} / L_{B}$ and as $\tau / \sigma$ becomes larger indicates that the interaction between nonlinearity and PMD is changed as the strength of the ellipticity is increased.

In conclusion, we have described the dependence of both the polarization decorrelation length and the diffusion length on the amount of ellipticity present in an optical fiber. These results demonstrate in particular that the expression for the DGD in terms of the fiber correlation length does not depend on the strength of ellipticity. However, the diffusion is in general anisotropic on the Poincaré sphere, which affects nonlinear interactions. A small ellipticity does not significantly affect results that are predicted by a model that assumes that fibers are linearly birefringent.

\section{References}

1. P. K. A. Wai and C. R. Menyuk, "Polarization mode dispersion, decorrelation, and diffusion in optical fibers with randomly varying birefringence," J. Lightwave Tech., Vol. 14, pp. 148-157, 1996.

2. N. Gisin, "Solutions of the dynamical equation for polarization dispersion," Opt. Commun, Vol. 86 , pp. 371-373, 1991.

3. A. Galtarossa, L. Palmieri, M. Schiano, T. Tambosso, "Statistical characterization of fiber random birefringence," Opt. Lett., Vol. 25, pp. 13221324, 2000.

4. R. Ulrich and A. Simon, "Polarization optics of twisted single-mode fibers," Appl. Optics, Vol. 18, pp. 2241-2251, 1979.

WJ6

5:30 PM

Second-Order Polarization-Mode Dispersion in Photonic Crystal Fibers

T. Larsen, A. Bjarklev, COM Research Center, Lyngby, Denmark; A. Peterson, J. Folkenberg, Crystal Fibre A/S, Birkerød, Denmark; P. Pehrson, Tellabs Copenhagen, Ballerup, Denmark,Email: ttl@com.dtu.dk.

We report the first experimental measurements of second-order Polarization-Mode Dispersion in two successive 900 meter pulls of a silica Photonic Crystal Fiber.

\section{Introduction}

Photonic Crystal Fibers (PCFs) form a new class of optical fibers, which have attracted significant attention during the last few years $[1,2,3]$. PCFs are silica fibers with a large number of air-holes located in the cladding region. The size and location of these air-holes provide a large degree of design flexibility, which has been used to tailor the optical properties and design large-mode area fibers [4], and dispersion tailored fibers [5]. Recently, PCFs with very low loss have been reported [6], which further strengthened the possibility of using PCFs for long-haul transmission. Another important parameter for long-haul transmission is Polarization-Mode Dispersion (PMD) PMD causes different polarizations to propagate with different velocities, which causes pulse broadening in a communication system [7]. PMD can be classified into first- and second-order PMD, where first-order PMD describes the Differential Group Delay (DGD) between the two orthogonal Principal States of Polarization (PSP) [8] and second-order PMD is the wavelength dependence of the PSP and the DGD, generally referred to as the rotation rate of the PSPs (2k) and the Polarizations dependent Chromatic Dispersion (PCD)[9]. A non-zero second-order PMD is caused by polarization mode-coupling and increases linearly with the fiber length, where first-order PMD increases with the square root of the fiber length [10]. Second-order PMD needs to be considered, since it decreases the effectiveness of PMD compensators [11], but can also decrease the system outage-probability in certain cases [12].

The first experimental measurements of firstorder PMD in PCFs was reported in [13], where the DGD was measured on three 100 meter silica PCFs with different core sizes. In this paper, we report what we believe is the first experimental measurements of second-order PMD in two 900meter successive pulls of PCFs, pulled under different conditions.

\section{Experiment}

The two studied PCFs had a triangular air-hole structure with a normalized air-hole size of $d /$ $\Lambda=0.43$. The background material was fluorinedoped silica, which lowered the refractive index of both the core and cladding material with $1.1 * 10^{-3}$ with respect to pure silica. Two 900 meters fibers, referred to as PCF1 and PCF2, was drawn from the same preform but under different pulling conditions. These fibers were coiled on two $160 \mathrm{~mm}$ diameter spools and spliced at both ends with a 1 meter pigtail with $\mathrm{FC} / \mathrm{PC}$ connectors. A microscope picture of the two fibers at the beginning of each pull is shown on Figure 1, and it shows an increased air-hole size of PCF2, compared to PCF1. The air-hole size of PCF1 and PCF2 was $1.46 \mu \mathrm{m}$ and $1.52 \mu \mathrm{m}$, respectively. The eccentricity of the cores was quantified using the formula: $e=1-d_{\text {mir }} / d_{\text {max }}$, where $e$ is the eccentricity and $d_{\min }$ and $d_{\max }$ is the minimum and maximum distances between two diagonal air-holes surrounding the core. The eccentricity of the cores of PCF1 and PCF 2 was 0.014 and 0.017 , respectively, indicating a slightly more eccentric core in PCF2 than in PCF1.

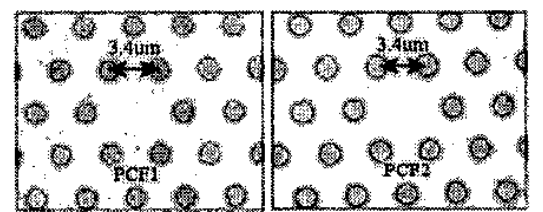

Figure 1. Microscope picture of the end facet of the two fiber samples.

The Jones matrix of each fiber was measured from $1530 \mathrm{~nm}$ to $1560 \mathrm{~nm}$ with $0.25 \mathrm{~nm}$ stepsize for PCF1 and 0.1 nm stepsize for PCF2. The DGD and the PSP was calculated using the Jones Matrix Eigenanalysis [14], and the two secondorder parameters, the $\mathrm{PCD}$ and the rotation rate of the PSPs (2k), was calculated from the wavelength derivative of the DGD and the PSP vector, respectively.

\section{Results and Discussion}

The measured first- and second-order PMD parameters for PCF1 and PCF2 are shown on Fig ure 2 and Figure 3, respectively. Due to the noisy DGD curve, the DGD data was lowpass filtered using a $10^{\text {th }}$ order Hamming window before the PCD was calculated. Since the noise only occurred on the DGD data, the noise was attributed to wavelength inaccuracy of the laser used in the experiment. As seen from the top curves of Figure 2 and Figure 3, there is a strong correlation between the PSP rotation rate (2k) and the DGD, which has also been observed in standard singlemode fibers [10].
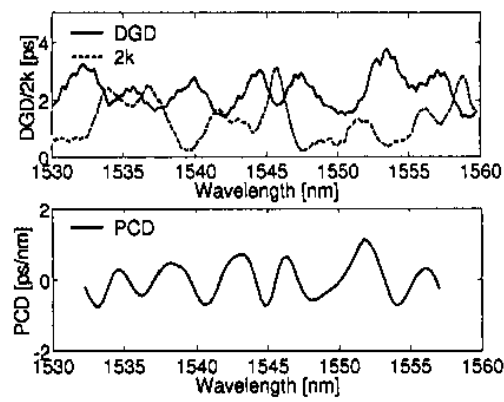

Figure 2. DGD, PCD and 2k measured on PCF1.
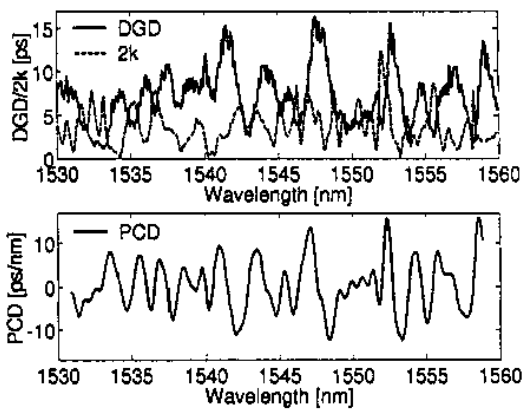

Figure 3. DGD, PCD and $2 \mathrm{k}$ measured on PCF2.

Figure 4 and Figure 5 shows the measured Probability Distribution Function (PDF) of the DGD of $P C F 1$ and PCF2, respectively. The dashed line indicates the Maxwell distribution for the theoretical DGD in the strong mode-coupling regime. The PMD of PCF1 and PCF2 were measured to $2.3 \mathrm{ps}$ and $7.1 \mathrm{ps}$, respectively. The PMD was also measured using the interferometric method and yielded a PMD of $2.1 \mathrm{ps}$ and $6 \mathrm{ps}$, respectively. These values were measured using an $80 \mathrm{~nm}$ broadband LED centred around $1550 \mathrm{~nm}$. These values are comparable to standard single-mode fiber values reported in the mid-1990's [15]. It should also be mentioned that the PMD around $1300 \mathrm{~nm}$ was measured to $0.9 \mathrm{ps}$ and $2.2 \mathrm{ps}$, respectively, and thereby indicating strong wavelength dependence of the PMD. The PDF of the DGD clearly indicates that $P C F 2$ is closer to the strong mode-coupling regime than $\mathrm{PCFl}$, even though PCF2 has 3 times higher PMD than PCF1. This indicates that the birefringence is increased and the mode-coupling length is decreased in PCF2 compared to PCF 1 .

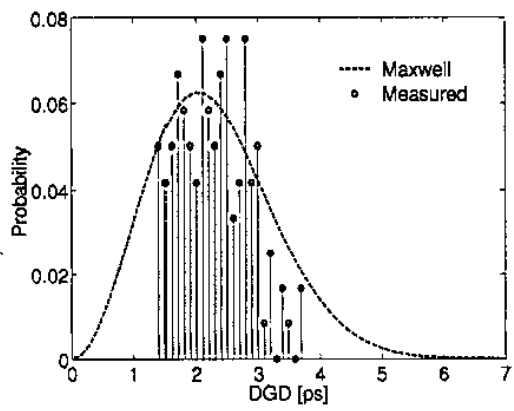

Figure 4. Probability Distribution Function of the DGD measured on PCF 1 . 


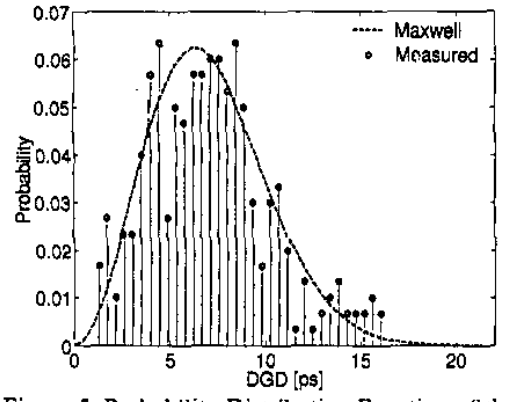

Figure 5. Probability Distribution Function of the DGD measured on PCF2.

\section{Conclusion}

We have successfully measured first- and secondorder PMD on two successive pulls of a triangular structured PCF. The two PCFs were pulled under different conditions, which affected both the birefringence and the mode-coupling length. The experiment showed that the PMD of these fibers behaved in the same way as in standard fibers and could be treated using conventional methods. The reported PMD was comparable to the PMD reported in the mid-1990s for standard fibers.

\section{References}

1. J. C. Knight et al., Opt. Lett, 21, p. 1547, 1996. 2. J. Broeng et al., Inv. Paper, Opt. Fiber. Tech. 5 p. $305,1999$.

3. T. M. Monro et al., Opt. Lett., 25, p. 206, 2000.

4. M. D. Dybendal, ECOC 2002, Tu3.4.2.

5. A. Bjarklev et al., ECOC 1998.

6. L. Farr et al., ECOC 2002, PD1.3.

7. C. D. Poole et al., IEEE Phot. Tech. Lett., Vol. 3 , No. 1, p. 68,1991 .

8. C. D. Poole and R. E. Wagner, Elec. Lett., Vol. 22, No. 19, 1986.

9. F. Bruyere, Opt. Fiber Tech., Vol. 2, No. 3, p. $269,1996$.

10. L. Nelson et al., IEEE Phot. Tech. Lett,, Vol. 11, No. 12, p. 1614, 1999.

11. H. Sunnerud et al, Jour. of Ligth. Tech., Vol. 20 , No. 3, p. 368, 2002.

12. A. O. Lima et al, ECOC 2002, Th7.1.2.

13. T. Niemi et al., ECOC 2002, Mol.9.

14. B. Heffner, IEEE Phot. Tech. Lett., Vol. 13, No. 7, p. $814,1993$.

15. M. C. de Lignie, Journ. of. Lightw. Tech., Vol 12 , No. 8, p. 1325,1994

\section{WK \\ 4:00 PM - 5:45 PM B308-B309}

\section{Amplifier Design and Characterization 1}

Jean-Marc Delavaux, Keopsys Inc., USA, Presider

WK1 (Invited) 4:00 PM

Advanced Amplifier Design: Physics and Systems Limitations

$\mathrm{K}$. Wundke, Corning Incorporated, Corning, NY, Email: wundkek@corning.com.

We discuss the design requirements of transientgain-controlled Erbium-doped fiber amplifiers for next generation photonic networks, possible design implementation and trade-offs.

\section{Introduction}

In today's modern photonic networks optical amplifiers are used as line amplifiers between fiber spans, as pre- and booster amplifiers, as loss compensators in OADM nodes, and as power equalizers, to name only some of the possible
Conventional

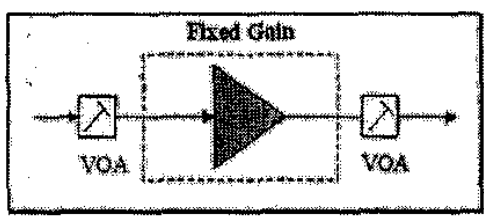

Conventional

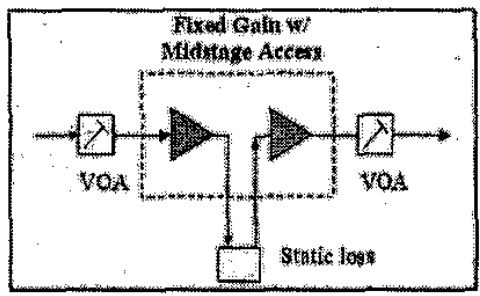

Emerging

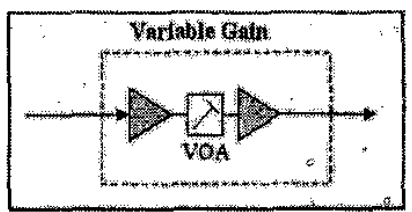

Next Gereration

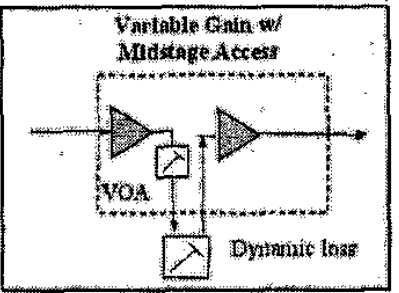

Fig. 1. Conventional fixed gain amplifier design vs. emerging variable gain amplifier design and next generation amplifier function, which exhibits variable gain, configurable midstage access and fast transient control.

applications. Each application drives a different set of design requirements in terms of performance; i.e. optical, electrical, and thermal; size and cost. In the past, much of the development effort was geared towards meeting specific performance targets with a minimum impact on cost and size. This approach was mainly driven by the fact that most amplifiers were to be used in static point-to-point long-haul and ultra long-haul transmission systems. Important design parameters for amplifiers used in such systems are OSNR, launch power, bandwidth, wideband gain flatness, gain tilt control and midstage access, where the latter is used to accommodate static high-loss components such as dispersion-compensating modules (DCM's). As a result, technology improvements were focused on parameters such as adding bandwidth [1], controlling tilt more efficiently $[2,3]$, reducing systematic wideband gain ripple $[4]$ and increasing reach by lowering the OSNR penaities [5].

The recent trend to reconfigurable long-haul network architectures, to reduce cost and improve system performance, already requires a shift in the development focus for optical amplifiers. Even more important, however, is the growing demand for the deployment of higher bandwidth services closer to the end customer, which means a new class of amplifiers for regional, metro-core and metro-access networks. The requirements for Erbium-doped fiber (EDF) amplifiers for such emerging network architectures are very different from the above. These networks demand tolerance for a much wider dynamic range of channel load and launch powers, rapid and accurate network reconfiguration without traffic interruption, new protection schemes, and new and more com plex loss elements such as dynamic DCM's and optical add/drop multiplexers (OADM's). Moreover, service providers benefit from these new features only if they are accompanied by much higher package density and lower overall installation and operating costs.

\section{Next generation EDF amplifier require-} ments

Reconfiguration and pay-as-you-grow upgrading of a network could cause an amplifier that today sees only a single channel to be used for as many as 100 and more channels in the future. This wide range of number of channel count together with the wide variation in fiber span lengths and the use of high, dynamic loss components yields input power ranges of up to $40 \mathrm{~dB}$ as well as gain variability of at least $15 \mathrm{~dB}$.

Additionally, ultra-fast and accurate transient control [6] becomes increasingly important as networks migrate to reconfigurability it prevents large surviving-channel gain excursions during channel-add and -drop events. A potential decrease in the surviving-channel gain during such a transient event causes performance degradation due to eye-closure and OSNR degradation at the receiver side, whereas an increase in the surviving-channel gain can build-up throughout the network and potentially damage other line components and receivers [7]

Another important requirement is the need for truly configurable midstage loss compensation to enable the use of a wider variety of loss components such as a static DCM with a fixed time delay or a dynamically OADM with a time dependent loss profile.

So far, the discussed design requirements are only focused on adding or improving performance, but in todays environment package size and density as well as total power efficiency are equally important. For example, amplifiers have to be able to fit on a card pitch less than $25 \mathrm{~mm}$, which also needs to incorporate the thermal dissipation scheme as well. Package density will aiso be increased by adding more functionality onto the same card. For example, optical performance monitoring and transceivers are to be found in the same footprint which was formerly reserved for the amplifiers alone.

3. Next generation EDF design considerations In contrast to the next generation EDF amplifier requirements, conventional EDF amplifiers have limited dynamic operating ranges and transient capability. Therefore, system engineers have had to place variable optical attenuators (VOA's) in front and after the amplifier, as shown on the left hand side in Fig. 1, to buffer the increased variablity. This causes severe OSNR and optical power penalties as well as significantly lower wall-plug efficiencies, increased size and added cost. Also, system vendors had to merge the VOA and the amplifier controls together on a higher level, which further increases complexity and cost and reduces overall performance.

Recently emerging controlled amplifier designs, as shown in Fig. 1 as well, partially circumvent that problem by providing a much wider dynamic gain and power range. Fig.2 shows the Noise Figure benefit of such a variable gain amplifier [8] as a function of optical gain compared to a conventional fixed gain amplifier. However, these designs still require to place two amplifiers back-to-back to compensate for high loss components. Additionally, the performance optimization that balances the two amplifiers and the midstage loss must be implemented at a higher system level, which intrinsically limits the overall performance.

Furthermore, for the transient controller to operate most effectively, its performance parameters mus match the requirements dictated by the latest generation of high-speed optical switches, which are able to initiate adds and drops with rise- and fall times of as little as $1 \mu$ s and power changes of at least $15 \mathrm{~dB}$. Moreover, power spikes due to techni- 